\title{
The Speed of Sound in Hadronic Matter
}

\author{
P. Castorina ${ }^{1}$, J. Cleymans ${ }^{2}$, D. E. Miller ${ }^{3,4}$ and H. Satz ${ }^{3}$ \\ 1 Dipartimento di Fisica, Università di Catania and INFN Sezione di Catania \\ I-95123 Catania, Italy \\ 2 Physics Department, University of Cape Town, South Africa, \\ 3 Fakultät für Physik, Universität Bielefeld, D-33501 Bielefeld, Germany \\ 4 Department of Physics, Pennsylvania State University \\ Hazleton Campus, Hazleton, PA 18202 USA
}

\begin{abstract}
:
We calculate the speed of sound $c_{s}$ in an ideal gas of resonances, whose mass spectrum is assumed to have the Hagedorn form $\rho(m) \sim m^{-a} \exp \{b m\}$, which leads to singular behavior at the critical temperature $T_{c}=1 / b$. With $a=4$ the pressure and the energy density remain finite at $T_{c}$, while the specific heat diverges there. As a function of the temperature, the corresponding speed of sound initially increases similarly to that of an ideal pion gas, until near $T_{c}$ resonance effects dominate, causing $c_{s}$ to vanish as $\left(T_{c}-T\right)^{1 / 4}$. In order to compare this result to the physical resonance gas models, we introduce an upper cut-off $M$ in the resonance mass integration. Although the truncated form still decreases somewhat in the region around $T_{c}$, the actual critical behavior in these models is no longer present.
\end{abstract}




\section{Introduction}

The abundant formation of resonances of increasing mass and rotational degrees of freedom is one of the most striking features of strong interaction physics, which has attracted intense theoretical attention for half a century or more. Even before the quark infrastructure of hadrons was known, a self-similar composition scheme, the statistical bootstrap model (SBM), led to an exponentially increasing resonance spectrum [1]. Shortly thereafter the dual resonance model (DRM) provided a description of hadron interactions in terms of the Regge resonance structure in the different kinematic channels [2], which again produced an exponentially increasing spectrum [3]. In both these cases the basic feature is the underlying partition structure [4-6], leading to the form

$$
\rho(m) \sim m^{-a} \exp b m,
$$

for the number of states (degeneracy) of resonances of mass $m$. This situation posed for the theory the two basic questions: what is the origin of so many resonances, and what is their effect on strong interaction thermodynamics?

The first question was answered by the advent of the quark model and of quantum chromodynamics (QCD), whereby the resonances are the different possible excitation states of the (initially) three quark flavors, together with their antiquarks. The second one was answered by R. Hagedorn [1], who showed that an exponentially rising resonance mass spectrum leads to an upper bound on the temperature of hadronic matter, $T_{c}=1 / b$, with $b$ as specified in the eq. (11). Beyond a certain point an increase in the energy density of the medium does not increase its temperature; instead, more and higher mass resonances are formed. The behavior of the medium at the "Hagedorn" temperature $T_{c}$ is determined by the power $a$ in eq. (1). In particular, it is shown that for $a \geq 4$, the energy density itself remains finite at $T_{c}$, only higher derivatives diverge there. The general structure of the resulting thermodynamics near $T_{c}$ is thus that of critical behavior, associated with a phase transition leading to a new state of matter, which we today take to be the quark-gluon plasma (QGP) [7].

The aim of this work is to determine in some detail the onset of the critical behavior in strongly interacting matter, when coming from the confined hadronic side. The system here is an interacting hadron system; but it is known that if the interaction is resonance dominated, the medium of interacting elementary particles can be replaced by an ideal gas of all possible resonances $[8,9]$. If we consider systems of vanishing overall baryon number density, the assumption of resonance dominance appears to be well satisfied; only for high baryon density do non-resonant repulsive forces come into play [10].

We shall therefore consider here an ideal resonance gas of vanishing overall baryon density, and study in particular the temperature dependence of the speed of sound, $c_{s}(T)$, in such a medium. By definition,

$$
c_{s}^{2}(T)=\left(\frac{\partial P}{\partial \varepsilon}\right)_{V}=\frac{s(T)}{C_{V}(T)},
$$

where $s=(\partial P / \partial T)_{V}$ is the entropy density and

$$
C_{V}(T)=\left(\frac{\partial \varepsilon}{\partial T}\right)_{V}
$$


is the specific heat at constant volume. If the resonance gas has a suitable degeneracy structure of the Hagedorn/DRM type, the specific heat diverges at the limiting temperature $T_{c}$, while the entropy density remains finite there. As a result, the speed of sound will vanish at $T_{c}$. It is thus expected that $c_{s}(T)$ is at low temperature $T$ that of an ideal gas of ground state hadrons ("pions"), until $T$ becomes high enough to bring resonance effects into play and eventually drive the system towards critical behavior.

The speed of sound has long been considered as a sensitive indicator of critical behavior in strongly interacting matter. Early lattice studies indeed found the expected sharp dip of $c_{s}^{2}$ in the critical region [11,12]. Later, more precise studies seem to indicate a weaker dip [13], in accord with the expectation that the deconfinement transition is a rapid cross-over, rather than a genuine phase transition with singular behavior of thermal observables. The success of physical resonance gas models in the prediction of hadron abundances [14] provided the stimulus to also calculate the speed of sound in such schemes, using a grand canonical partition function of an ideal gas of all experimentally observed states up to a certain large mass. Again one finds a decrease of $c_{s}^{2}(T)$ as the temperature is brought near the expected critical point $[13,15]$.

In the next section we will evaluate the thermodynamical functions, first for an ideal gas of pions and then for an ideal resonance gas with an exponentially growing mass spectrum of the form (1). We shall refer to such a system as a Hagedorn resonance gas. Following this, we calculate the resulting speed of sound and study in detail how is approaches the expected critical behavior. In the final section, we compare our results to those obtained for the physical resonance gas based on the actually observed resonances up to a mass limit of $2.5 \mathrm{GeV}$ [16], as well as to the results from studies in finite temperature lattice QCD.

\section{Thermodynamics of a Hagedorn Gas}

For an ideal Boltzmann gas of identical scalar particles of mass $m_{0}$ and three charge states ("pions") contained in a volume $V$, the grand partition function is defined as

$$
Z(T, V)=\sum_{N} \frac{1}{N !}\left[\frac{3 V}{(2 \pi)^{3}} \int d^{3} p \exp \left\{-\sqrt{p^{2}+m_{0}^{2}} / T\right\}\right]^{N} .
$$

This expression can be evaluated, giving

$$
\ln Z(T, V)=3 \frac{V T m_{0}^{2}}{2 \pi^{2}} K_{2}\left(m_{0} / T\right)
$$

where $K_{2}(x)$ is the modified Bessel function of the second kind. From this equation we obtain

$$
P_{0}(T)=\left(\frac{\partial \ln Z}{\partial V}\right)_{T}=\frac{3 m_{0}^{2} T^{2}}{2 \pi^{2}} K_{2}\left(m_{0} / T\right)
$$

for the pressure and

$$
\varepsilon_{0}(T)=T^{2}\left(\frac{\partial \ln Z}{\partial T}\right)_{V}=\frac{3 m_{0}^{2} T^{2}}{2 \pi^{2}}\left[3 K_{2}\left(m_{0} / T\right)+\left(\frac{m_{0}}{T}\right) K_{1}\left(m_{0} / T\right)\right]
$$

for the energy density. Using these relations,

$$
s_{0}(T)=\frac{\varepsilon_{0}(T)+P_{0}(T)}{T}=\frac{3 m_{0}^{2} T}{2 \pi^{2}}\left[4 K_{2}\left(m_{0} / T\right)+\left(\frac{m_{0}}{T}\right) K_{1}\left(m_{0} / T\right)\right]
$$


provides the entropy density.

We now extend this to an ideal Boltzmann gas of resonances, described by an exponentially increasing mass spectrum of the Hagedorn form (1). For the full spectrum of hadrons, including ground state pions as well the resonances, we thus write

$$
\rho(m)=3 \delta\left(m-m_{0}\right)+A m^{-4} \exp \left\{m / T_{c}\right\} \theta\left(m-2 m_{0}\right) .
$$

Here the constant $A$ (of dimension $\mathrm{m}^{3}$ ) provides the normalization of the resonance contributions relative to that of the pions, and we have chosen the power term $a=4$ in order to obtain second order singular behavior, as will be shown shortly. The $\theta$-function assures that the resonance spectrum starts above the two-pion threshold. With the spectrum (9), the logarithm of the grand canonical partition function is now given by

$$
\ln Z(T, V)=\frac{V T}{2 \pi^{2}}\left\{3 m_{0}^{2} K_{2}\left(m_{0} / T\right)+A \int_{2 m_{0}}^{\infty} d m m^{-2} \exp \left\{m / T_{c}\right\} K_{2}(m / T)\right\} ;
$$

the pressure thus becomes

$$
P(T)=P_{0}(T)+\frac{A T^{2}}{2 \pi^{2}} \int_{2 m_{0}}^{\infty} d m m^{-2} \exp \left\{m / T_{c}\right\} K_{2}(m / T)
$$

with the pion gas pressure $P_{0}(T)$ given by eq. (6) . Similarly, we obtain

$$
\varepsilon(T)=\varepsilon_{0}(T)+\frac{A T^{2}}{2 \pi^{2}} \int_{2 m_{0}}^{\infty} d m m^{-2} \exp \left\{m / T_{c}\right\}\left[3 K_{2}(m / T)+\left(\frac{m}{T}\right) K_{1}(m / T)\right],
$$

for the energy density and

$$
s(T)=s_{0}(T)+\frac{A T}{2 \pi^{2}} \int_{2 m_{0}}^{\infty} d m m^{-2} \exp \left\{m / T_{c}\right\}\left[4 K_{2}(m / T)+\left(\frac{m}{T}\right) K_{1}(m / T)\right] .
$$

for the entropy density; here $\varepsilon_{0}(T)$ and $s_{0}(T)$ are the corresponding pion gas quantities (7) and (8).

The Bessel functions appearing in these relations have the asymptotic form for large argument

$$
K_{n}(m / T)=\sqrt{\frac{\pi T}{2 m}} \exp \{-m / T\}[1+O(T / m)] .
$$

This shows that the integrals determining pressure, energy and entropy density all converge for for $T \leq T_{c}$, while diverging for $T>T_{c}$. The convergence of the thermodynamics potentials at $T=T_{c}$ is a direct consequence of our choice $a=4$ in the resonance spectrum; for $a \leq 7 / 2$, both energy and entropy density would diverge there. In our case, one further temperature derivative is needed to cause a divergence, leading to a diverging specific heat at $T_{c}$. We shall return to the detailed form shortly.

Given the analytical form (9) of the resonance spectrum, both $\varepsilon_{0}(T)$ and $\varepsilon(T)$ can be evaluated numerically. For this, the resonance weight $A$ has to be specified. With $\varepsilon\left(T_{c}\right) / T_{c}^{4} \simeq 5$, as obtained in lattice studies for the case of two quark flavors [17], we obtain $A=15.35 T_{c}^{3}$. Using this value, we get the energy density behavior of the ideal resonance gas shown in Fig. 1, where it is compared to that of the ideal pion gas. We see that around $T / T_{c} \simeq 0.6$, resonances come significantly into play, so that $\varepsilon(T)$ begins to increase above the pion gas value, until at $T_{c}$, resonances provide the dominant part of the energy density. 


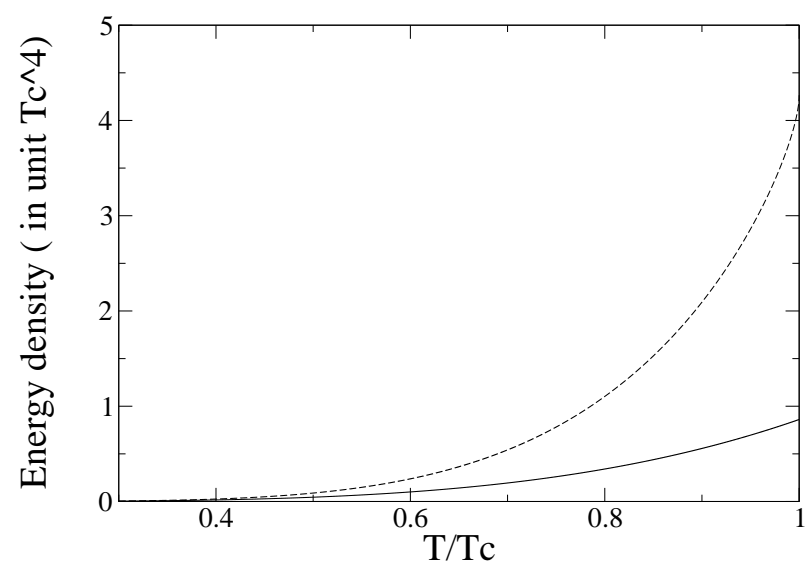

Figure 1: Energy density vs. temperature for an ideal pion gas (solid lower curve) and an ideal resonance gas (dashed upper curve)

\section{The Speed of Sound in a Hagedorn Gas}

The speed of sound $c_{s}$ at constant volume is defined by eq. (2). For the case of the ideal pion gas we obtain from the eqs. (3) and (12)

$$
C_{V}^{0}(T)=3 s_{0}(T)+\frac{3 m_{0}^{4}}{2 \pi^{2} T} K_{2}\left(m_{0} / T\right) .
$$

Together with the eqs. (2) and (8) this leads to

$$
\frac{1}{c_{s}^{2}}-3=\frac{3 m_{0}^{4} K_{2}\left(m_{0} / T\right)}{2 \pi^{2} T s_{0}}=\frac{m_{0}^{2} K_{2}\left(m_{0} / T\right)}{4 T^{2} K_{2}\left(m_{0} / T\right)+m_{0} T K_{1}\left(m_{0} / T\right)}
$$

for the ideal pion gas. Making use of the large argument limit (14) of the Bessel functions, we find for $T \rightarrow 0$, the squared speed of sound $c_{s}^{2}$ vanishes linearly with $T$. The small argument limit

$$
K_{n}(x)=\frac{2^{n-1}(n-1) !}{x^{n}}\left[1-O\left(x^{2}\right)\right]
$$

shows that for $T \rightarrow \infty$, we get $c_{s}^{2} \rightarrow 1 / 3$. The overall behavior below $T_{c}$ is shown in Fig. 2. For the Hagedorn gas, the same line of argument gives

$$
C_{V}(T)=3 s(T)+\frac{1}{2 \pi^{2} T}\left[3 m_{0}^{4} K_{2}\left(m_{0} / T\right)+A \int_{2 m_{0}}^{\infty} d m \exp \left\{m / T_{c}\right\} K_{2}(m / T)\right]
$$

for the specific heat. As $T \rightarrow T_{c}$, the entropy density remains finite. However, the integral in eq. (18) diverges so that at the Hagedorn temperature we have the critical behavior given as

$$
C_{V}(T) \sim\left(T_{c}-T\right)^{-\alpha},
$$

whereby the resulting critical exponent was found to be $\alpha=1 / 2[5]$.

An immediate further consequence of the diverging specific heat is that now the speed of sound vanishes at $T_{c}$. To obtain the functional form of this behavior, we make use of eqs. (13), (2) and (18) to get

$$
\frac{1}{c_{s}^{2}}-3=\frac{3 m_{0}^{4} K_{2}\left(m_{0} / T\right)+A \int_{2 m_{0}}^{\infty} d m \exp \left\{m / T_{c}\right\} K_{2}(m / T)}{2 \pi^{2} T s_{0}+A T \int_{2 m_{0}}^{\infty} d m m^{-2} \exp \left\{m / T_{c}\right\}\left[4 T K_{2}(m / T)+m K_{1}(m / T)\right]}
$$




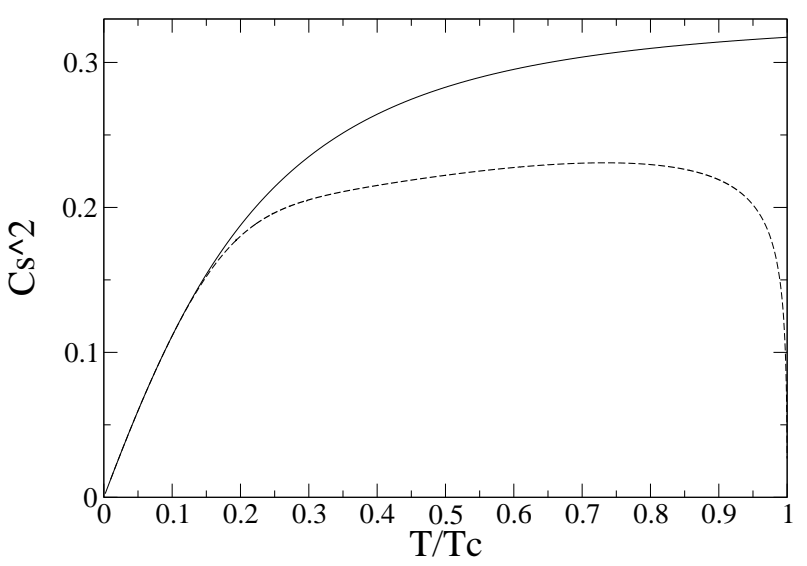

Figure 2: Speed of sound vs. temperature for an ideal pion gas (solid upper curve) and a Hagedorn gas (dashed lower curve)

for the behavior $c_{s}^{2}$ in the Hagedorn gas. In this relation, the second term in the numerator diverges as $T \rightarrow T_{c}$, which in turn causes the speed of sound to vanish there.

Making use of the value of $A$ obtained above, we show in Fig. 2 the Hagedorn gas result compared to the ideal pion gas form. It is seen that the Hagedorn gas follows the pion gas form closely until about $T / T_{c} \simeq 0.2$; from then on, any further increase of the average energy density is mainly turned into resonance masses, keeping the speed of sound roughly constant but non-zero. Only for temperatures very close to the critical point, for $T / T_{c} \geq 0.9$, do we reach a regime in which more and more of the energy goes into forming massive resonances. The truly critical region of the Hagedorn gas is thus restricted to a rather narrow band below the critical temperature. Magnifying this region, we show in Fig. 3 the behavior of $c_{s}^{2}$ as function of the expected critical form $\left(T_{c}-T\right)^{1 / 2}$. It is seen that only in the last $1-2 \%$ below $T_{c}$ the predicted behavior actually occurs.

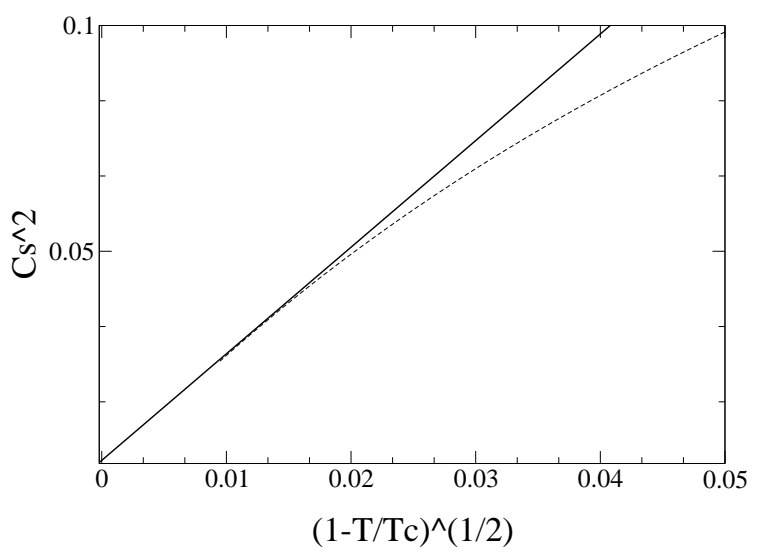

Figure 3: Speed of sound of a Hagedorn gas (dashed lower curve) in the critical region, compared to the predicted critical behavior $\left(T_{c}-T\right)^{1 / 2}$ (solid upper curve)

We can obtain further information on the extent of the critical region by making use of the 
interaction measure

$$
\Delta(T) \equiv \frac{\epsilon(T)-3 P(T)}{T^{4}}
$$

formed from the trace of the energy-momentum tensor; it vanishes for an ideal gas of massless constituents. In Fig. 4, we show the modifications coming in for a resonance gas. Again we notice a rather large region of "normal" resonance effects, until very close to $T_{c}$, the critical rise sets in leading to a diverging slope of $\Delta(T)$ as $T \rightarrow T_{c}$.

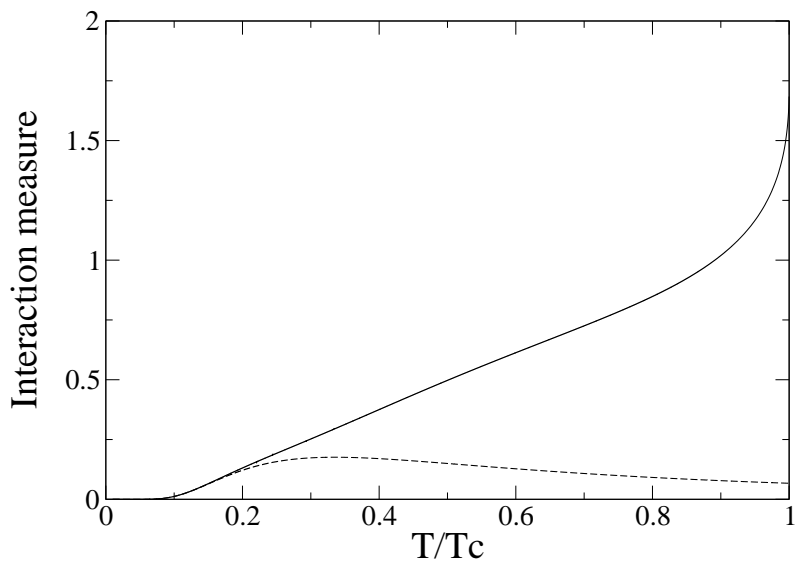

Figure 4: Interaction measure for an ideal pion gas (bottom curve) compared to that of a Hagedorn gas (top curve)

\section{The Low Mass Resonance Spectrum}

In this section we want to consider in some more detail the effect of the resonance spectrum form in the low mass range on the speed of sound $c_{s}(T)$. Our choice of the power factor of $\rho(m)$ in eq. (11) was dictated by the desired behavior of the thermodynamic observables at the divergence point $T_{c}$. When $a=4$, we had a finite energy density and a singular specific heat. The crucial feature for this was, however, the behavior at large $m$, for which a change in the threshold form at $m=2 m_{0}$ would not modify that property. The functional form for the spectrum (1) is shown in Fig. 5. It has a minimum at $m=4 / b$, before it begins its exponential rise. Thus it is evident that this minimum has no real physical meaning and can be replaced by a more reasonable threshold behavior of the resonance contributions. Therefore, we can replace $\rho(m)$ in (1) by

$$
\rho_{s}(m)=\left[1-\left(\frac{2 m_{0}}{m}\right)^{s}\right] m^{-4} \exp \{b m\}
$$

with some integer constant power $s>0$. Clearly $\rho_{s}(m)$ still retains the same critical behavior. The spectral form produced by (22) for $s=2$ is included in Fig. 5. Since the region of the low mass resonances contributes very little to the functional form of the speed of sound, the changes that such a threshold factor produces in $c_{s}^{2}(T)$ are effectively negligible. 


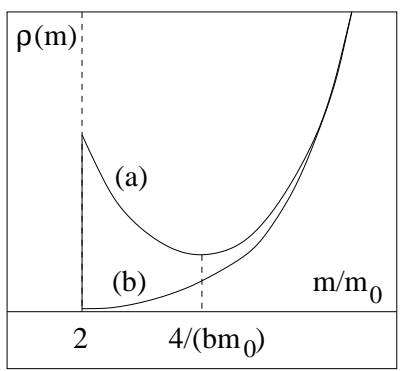

Figure 5: Resonance spectrum for eq. (1) with $a=4$, compared to the form given by eq. (22).

\section{The Physical Resonance Gas}

We now want to compare our results to those obtained in studies of an ideal (Boltzmann) gas of actual physical resonances. The relevant partition function thus becomes the sum over all states listed by the Particle Data Compilation [16] up to some mass value of $M$, above which the experimental information is too incomplete to be of use. Typically one has $M=2-3 \mathrm{GeV}$. For each state of mass $m_{i}$, we include a degeneracy $g_{i}$ determined by the spin, isospin, baryon number and strangeness degrees of freedom. The partition function is then given by

$$
\ln Z_{P}(T, V)=\sum_{\mathrm{i}} \ln Z_{P}^{i}(T, V)
$$

with

$$
\ln Z_{P}^{i}(T, V)=\frac{g_{i}}{(2 \pi)^{2}} \int d^{3} p \exp \left\{-\frac{\sqrt{p^{2}+m_{i}^{2}}}{T}\right\}=g_{i} \frac{V T}{2 \pi^{2}} m_{i}^{2} K_{2}\left(m_{i} / T\right),
$$

specifying the contribution of a resonance of $m_{i}$.

Before we calculate the speed of sound for such a "physical" resonance gas, let us see what effect a mass truncation in the resonance integration has for the Hagedorn gas. We thus consider the form obtained if we replace in eq. (10) the integral over $\left[2 m_{0}, \infty\right]$ by one over $\left[2 m_{0}, M\right]$. The results are shown in Fig. 6 for several values of $M$. Since the truncated system has no divergence at $T=T_{c}$, that is since the specific heat now remains finite there, we can continue the $c_{s}^{2}$ curves also to values above $T_{c}$. Eventually, for any finite $M$, they will converge to $c_{s}^{2}=1 / 3$.

We now turn to the physical resonance gas and calculate the speed of sound for several values of the upper limit $M$ in resonance mass. The results are shown in Fig. 7 , Qualitatively, they agree quite well to what was found for the truncated Hagedorn gas. It is seen that in both scenarios, the inclusion of further heavy resonances somewhat lowers the speed of sound in the temperature range $0 \leq T \leq 200 \mathrm{MeV}$, but it never produces a sharp minimum.

Futher inspection does show, however, a difference in the behavior of the two formulations. In Fig. 8, we show the speed of sound at $T=T_{c}$ in the Hagedorn gas for increasing resonance mass bounds. It is seen to converge to zero for $M \rightarrow \infty$, which means that it is only the mass bound which prevents critical behavior.

In the case of the physical resonance gas, we also have to consider the form of the degeneracy. In the degeneracy factor $g_{i}$, only the spin variable $J_{i}$ can result in an unbounded increase with $i$. For resonances on linear Regge trajectories, we have

$$
J_{i} \sim \alpha^{\prime} m_{i}^{2}
$$




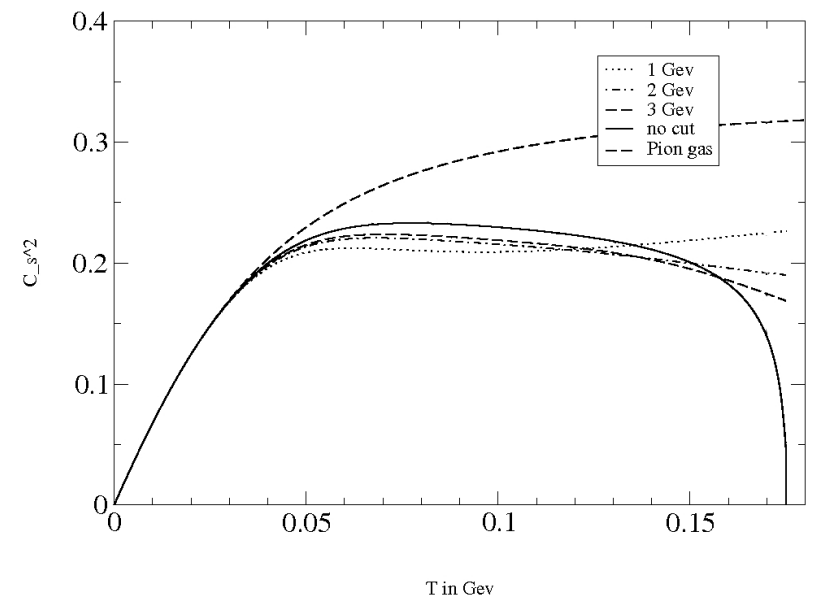

Figure 6: Speed of sound in a Hagedorn gas with resonance mass truncation, $m \leq M$.

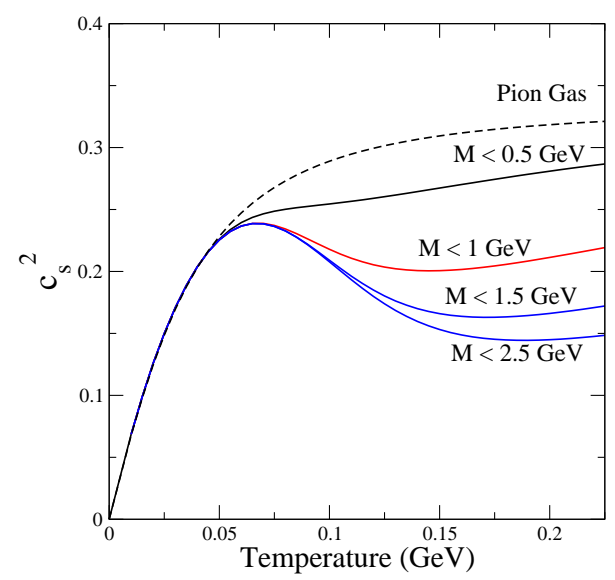

Figure 7: Speed of sound in a physical resonance gas with upper limits $M$ in resonance mass.

where $\alpha^{\prime} \simeq 1 \mathrm{GeV}^{-2}$ is the Regge resonance slope. Hence the spin degeneracy $\left(2 J_{i}+1\right)$ implies

$$
g\left(m_{i}\right) \sim m_{i}^{2}
$$

It is clear that with such a degeneracy, the partition function will remain analytic for all values of $T$, so that we will never encounter critical behavior, no matter what the upper bound on the mass is. This explains why, as seen in Fig 9, an increase of $M$ appears to produce a convergent finite value of $c_{s}^{2}\left(T_{c}\right)$.

Thus it is not only the upper limit in resonance mass which prevents the physical resonance gas from ever showing critical behavior. The crucial factor is the use of a degeneracy determined by quantum number degrees of freedom only. Such a degeneracy grows, as we saw above, as $m^{2}$, and as a consequence, the partition function always remains analytic. The critical behavior arising for a Hagedorn resonance gas is due to a degeneracy growing exponentially in $m$. This, in turn, is a consequence of counting as distinct degenerate states all partitions of a given resonance into all other possible resonances. As we noted above, both the SBM and the DRM obtain the exponential mass degeneracy from the corresponding partition problem. 


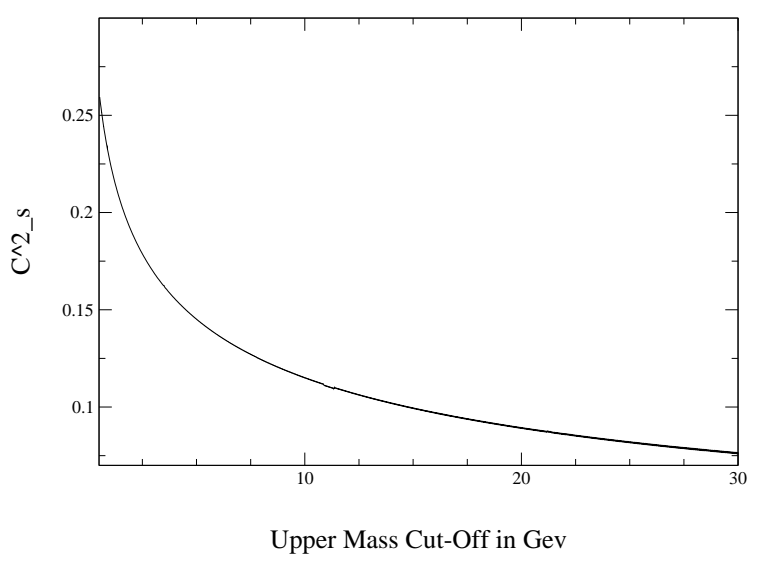

Figure 8: Speed of sound for a Hagedorn gas as function of the upper limit $M$ in resonance mass.

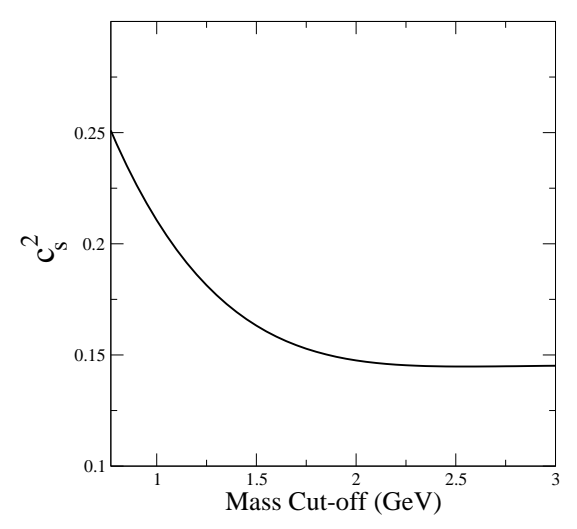

Figure 9: Speed of sound at $T=175 \mathrm{MeV}$ in a physical resonance gas, as function of upper resonance mass limit $M$.

Do these considerations have any physical implications? They clearly would, if deconfinement were an actual phase transition with a diverging specific heat. If it is only a rapid cross-over, or if the transition should lead to a finite specific heat at $T_{c}$ (such as produced in the $O(4)$ universality class), the behavior of the speed of sound obtained from the physical resonance gas even at $T_{c}$ can remain a good approximation.

On the other hand, we have also seen that the actual critical region in the Hagedorn gas is a very narrow band just below $T_{c}$. Up to a temperature of $T \simeq 0.95 T_{c}$, the observed behavior is due to the normal resonance pattern, and here physical resonance gas and Hagedorn gas can certainly agree. This leaves us with an amusing question: is the temperature obtained in abundance analyses of hadron species indeed the temperature of the confinement/deconfinement transition, or does the replacement of an interacting hadron gas by a physical resonance gas break down very close to a critical point?

Acknowledgements We would like to thank Jürgen Engels and Olaf Kaczmarek for their help with the evaluations. 


\section{References}

[1] R. Hagedorn, Supplemento al Nuovo Cimento, Volume III, 147 (1965);

Nuovo Cim. 56 A (1968) 1027

[2] G. Veneziano, Nuovo Cim. 57A (1968) 190.

[3] K. Bardakci and S. Mandelstam, Phys. Rev. 184 (1969) 1640;

S. Fubini and G. Veneziano, Nuovo Cim. 64A (1969) 811.

[4] K. Huang and S. Weinberg, Phys. Rev. Lett. 25 (1970) 895.

[5] H. Satz, Phys. Rev. D 19 (1979).

[6] Ph. Blanchard, S. Fortunato and H. Satz, Europ. Phys. J. C 34 (2004) 361.

[7] N. Cabibbo and G. Parisi, Phys. Lett. 59B (1975) 67.

[8] E. Beth and G. E. Uhlenbeck, Physica 4 (1937) 915.

[9] R. Dashen, S. Ma and H. J. Bernstein, Phys. Rev. 187 (1969) 345.

[10] P. Castorina, K. Redlich and H. Satz, Europ. Phys. J. C 59 (2009) 67.

[11] R. V. Gavai and A. Goksch, Phys. Rev. D 33 (1986) 614.

[12] K. Redlich and H. Satz, Phys. Rev. D 33 (1986) 3747.

[13] For later lattice studies, see F. Karsch, PoS C POD07 (2007) 026 [arXiv:hep-lat/0711.0656]

[14] J. Cleymans et al., Phys. Lett. B 242 (1990) 111;

J. Cleymans and H. Satz, Z. Phys. C 57 (1993) 135;

for a recent review, see

P. Braun-Munzinger, K. Redlich and J. Stachel, in Quark-Gluon Plasma 3, R. C. Hwa and X.-N. Wang (Eds.), World Scientific, Singapore, 2004.

[15] P. Braun-Munzinger and J. Stachel, arXiv:nucl-th/960617;

D. Prorok and L. Turko, arXiv:hep-ph/0101220.

[16] C. Amsler et al. (Particle Data Group), Phys. Lett. B 667 (2008) 1.

[17] F. Karsch, E. Laermann and A. Peikert, Phys. Lett. B 478 (2000) 447. 